\title{
Recherche d'une prévision précoce de l'amplitude des variations saisonnières du diamètre testiculaire et du pourcentage de spermatozoïdes anormaux chez le bélier Ile-de-France. 1. Animaux nés en février
}

\author{
G. COLAS, J. LEFEBVRE $\left({ }^{*}\right)$, J. GUÉRIN
}

Station de Physiologie de la Reproduction, I.N.R.A. Nouzilly, 37380 Monnaie, France.

$\left(^{*}\right)$ Laboratoire de Génétique Factorielle, I.N.R.A. Jouy-en-Josas, 78350, France.

Summary. Early prediction of the magnitude of seasonal variations in testis diameter and percentage of abnormal spermatozoa in the /le-de-France ram. 1. Lambs born in February.

The aim of this study, conducted in spring born animals, was to analyse if within a group of males of the same age, there is any relationship between the testis diameters (TD) or between the percentages of morphologically abnormal spermatozoa (AS) recorded in the same animal at two periods of its life (be it at two different spring periods or at two stages of its age : ram lamb and aduit).

Eighteen Ile-de-France ram lambs born in February 1980 and issued from five adult rams whose seasonal variations in TD and AS had been followed for two consecutive years were used in this study.

Semen was collected (artificial vagina, $1 \mathrm{ej} / \mathrm{s} / \mathrm{wk}$ ) from October 1980 until December 1983 (slaughter of the animals) every semester during "Periods" $(n=7)$. Within a same year, the first semester (February to June) was called «Spring», the second (August to December) «Autumn».

AS (150 cells/smear) were controlled on each ejaculate and the same week maximum TD of both testes was measured using a calliper.

Correlations were calculated for TD and AS between individual values (simple correlations) or between groups of individual values (multiple or canonic correlations) at different periods. These values were chosen as follows :

TD : 4th and 5th control for the first period ( $P 1$, ram lamb); the three lowest values recorded in April (P2, P4, P6) ; the three mean highest values recorded in Autumn (P3, P5, P7).

AS: the first five measurements in $\mathrm{P} 1$; the four highest percentages obtained in March-April in P2, P4, P6 (correlations were not calculated in autumn since AS were very few).

I $a$ : On the whole, TD increased with the age of the animal until 24-27 months and passed alternatively by minimal (February to April) and maximal (September) values each year. Testis growth due to the effect of photoperiodism started every year in the first days of June.

I b : Except alterations of semen quality in August 1983 (due to high local temperatures), AS were always higher in Spring than in Autumn, maximal values being reached in February (P4) or in March-April (P2 and P6). Their seasonal variations were thus opposed 
to those of TD ( $r=-0,671$ between weekly means of AS and TD of the whole group of animals from $\mathrm{P} 2$ to $\mathrm{P} 6$ )

//-TD : Individual TD of ram lambs were significantly correlated with those recorded in the same adult animal (P1-P2, P1-P6, but P1-P4 NS) especially in autumn (P1-P3, P1-P5, $\mathrm{P} 1-\mathrm{P} 7)$

Correlations were still higher when measurements were taken in the adult at one year intervals :

$P 2-P 4, P 2-P 6, P 4, P 6$, in spring

P3-P5, P3-P7, P5, P7, in autumn.

By contrast, when registered at 6-month intervals, relationships were lower and often no more significant (P2-P3, P2-P5, P2-P7).

$1 /-A S$ : In young animals (ram lamb $\mathrm{P} 1$ ), the semen quality was poorly related to that of the adult state. This relationship was small and rarely significant in the adult.

These results show that it is possible to carry out an early (ram lamb or 1 -year old lambs) preselection of the best sires, only based on testis development.

Chez le bélier, de nombreux travaux ont montré que les dimensions testiculaires (Ortavant, 1958 ; Pelletier, 1971 ; Lincoln, 1977 ; Schanbacher et Ford, 1979 ; Colas et al., 1986) et la qualité des spermatozoïdes éjaculés (Neves et al., 1980 ; Colas, 1980, 1981 ; Amir et al., 1986) sont sujettes à des variations saisonnières dont l'amplitude, très inégale selon les individus, traduit une plus ou moins grande sensibilité à la durée du jour. II serait intéressant de savoir si, chez un même animal, ces fluctuations apparaissent chaque année à la même époque et, dans l'affirmative de pouvoir les déceler de bonne heure, de préférence avant l'âge d'un an. On ignore, par ailleurs, si elles se transmettent dans la descendance d'un reproducteur. La littérature ne fournit aucun élément de réponse précis à ces questions.

Les variations saisonnières sont, en fait, la conséquence de l'action de la photopériode sur la fonction testiculaire (Ortavant et Thibault, 1956; Alberio, 1976). On sait aussi que chez le jeune, la croissance de la gonade (Courot, 1975 ; Alberio, 1976) et l'importance des réserves des cellules de Sertoli (de Reviers et al., 1980) dépendent de la saison de naissance, c'est-à-dire essentiellement de l'environnement lumineux naturel sous nos latitudes. II n'est donc pas impossible que des sujets issus de mêmes pères, mais nés à des époques différentes aient des performances sexuelles différentes. La prise en compte de la saison de naissance devenait alors un élément nécessaire dans ce type d'expérience.

Dans la présente analyse, nous avons essayé de dégager les relations qui peuvent exister au sein d'un même groupe de mâles nés au printemps, entre les diamètres testiculaires et aussi entre les proportions d'anomalies morphologiques enregistrés à différentes époques de leur vie. Une seconde étude, réalisée sur les mêmes mâles, portera sur la comparaison des performances (mêmes paramètres) des familles entre lesquelles se répartissent ces mâles. Une démarche identique, appliquée à des animaux nés en automne (même pères), fera l'objet d'une autre présentation. 


\section{Matériel et méthodes.}

1. Animaux expérimentaux. - Cinq béliers adultes $(A, B, C, D, E)$ inscrits au Livre Généalogique de la race lle-de-France ont eu 18 descendants nés en février 1980. Les observations effectuées sur ces 18 descendants ont débuté en octobre 1980 et se sont terminées en décembre 1983 lorsque tous les animaux ont été abattus sauf un, mort accidentellement quelques jours auparavant.

II. Collecte de sperme. - A l'âge d'environ 7 mois, les agneaux ont été dressés à la collecte de sperme (vagin artificiel) en présence d'une femelle en chaleur.

Les prélèvements de semence ont été effectués toutes les semaines (1 éj./ $/ \hbar / \mathrm{sem}$.), le même jour sur tous les animaux, de février à mai ou juin ("Printemps") et d'août, septembre ou octobre à novembre ou décembre ( Automne »). Ces époques de collecte sont appelées «Périodes" dans notre étude.

III. Contrôles. - Le poids corporel des animaux a été mesuré une fois par semaine pendant toute la durée de l'expérience (7 périodes) et le jour de l'abattage.

Le diamètre antéro-postérieur maximum (DT) des testicules (gonade + enveloppes) a été mesuré au millimètre près toutes les semaines ( 7 périodes) à l'aide d'un pied à coulisse. Ces mesures ont été réalisées chez tous les animaux par la même personne pendant les six premières périodes (et le jour de l'abattage) et par un autre opérateur au cours de la dernière période. Elles ont été complétées par la pesée des testicules et des épididymes aussitôt après l'abattage.

Les anomalies morphologiques (AM) étaient contrôlées chaque semaine sur un échantillon de 150 spermatozoïdes par éjaculat selon une méthode déjà décrite (Colas, 1980).

\section{Expression et analyse des résultats.}

- Expression graphique. L'évolution des moyennes hebdomadaires (DT, $A M)$ et de leur écart type pendant les 7 périodes est représentée sous forme graphique.

- Corrélations simples (CS), multiples (CM) et canoniques (CC). Pour savoir si les valeurs d'un même paramètre (DT, $A M$ ) mesuré à deux moments de la vie de l'animal (aut. 1980 et aut. 1981 ; print. 1981 et print. 1982, etc...) sont liées, il faut d'abord rechercher, pour chaque période, les dates où les moyennes hebdomadaires (DT, AM) sont les plus basses ou les plus élevées et calculer ensuite les corrélations entre les données individuelles enregistrées à ces dates.

Les calculs ont été effectués dans chaque groupe à partir des données individuelles prises 2 à 2 (CS) ou à partir de groupes de données (CC).

Les variations hebdomadaires du pourcentage des $A M$ étant plus fortes que celles du DT, en particulier au printemps (Colas et al., 1986), il s'est avéré nécessaire de prendre en considération un nombre plus important de données individuelles dans le cas des anomalies morphologiques. 
Les corrélations canoniques par étape $\left(\rho^{2}\right)$ ont été établies selon la méthode mise au point par Lefebvre (1976). Les valeurs figurant dans les tableaux 2 et 3 sont toujours les données maximales obtenues. Les chiffres indiqués entre parenthèses correspondent au numéro des moyennes dans l'ordre où elles figurent sur les graphiques 1 et 2 et où elles ont permis d'obtenir la corrélation la plus élevée.

Ex. : $\rho^{2}=0,88^{* *}(14 \& 15 \times 81 \& 80 ;$ tabl. 2$)$ signifie qu'il existe une corrélation canonique dont le carré est égal à 0,88 entre les deux groupes de données, celui des $14^{\mathrm{e}}$ et $15^{\mathrm{e}}$ points d'une part (Période $\mathrm{P} 2$, fig. 1 ) et celui des $81^{\mathrm{e}}$ et $80^{\mathrm{e}}$ points d'autre part (Période P6, fig. 1 ).

Pour deux groupes de deux facteurs chacun, les seuils de signification sont les suivants :

$\begin{array}{ccc} & \mathrm{n}=18 & \mathrm{n}=17 \\ \mathrm{P}<0,05: & 0,45 & 0,47 \\ \mathrm{P}<0,02: & 0,50 & 0,53 \\ \mathrm{P}<0,01: & 0,57 & 0,59\end{array}$

\section{Résultats.}

Au cours de la première période, un des jeunes béliers a refusé la collecte au vagin artificiel. Le calcul des pourcentages hebdomadaires moyens d'AM est donc établi pour un effectif de 17 mâles seulement en P1 et de 18 par la suite.

\section{Evolution générale des paramètres étudiés.}

A) Poids corporel - Le contrôle systématique du poids corporel a permis de confirmer que la croissance pondérale des animaux s'est déroulée de façon normale tout au long de l'expérience.

B) Diamètre testiculaire. - La figure 1 peut laisser supposer qu'au cours de la première période, le DT diminue de la $1^{\text {re }}$ (S1) à la $4^{\mathrm{e}}$ semaine (S4) et augmente ensuite jusqu'au moment du dernier contrôle (S7). L'analyse statistique ne révèle en fait aucune différence significative entre ces trois groupes de données (S1, S4, S7).

A partir de l'âge d'un an, le DT augmente avec l'âge, en passant alternativement par des minima (février à avril) (P2, P4, P6) et des maxima (septembre) (P3, P5, P7).

Au printemps, de 1981 à 1983, les valeurs relevées au mois de mars à mai ne sont jamais supérieures $(P>0,05)$ à celles obtenues chaque année lors du premier contrôle de février. Il faut attendre les tout premiers jours de juin pour enregistrer une différence significative entre mai et juin, c'est-à-dire pour voir apparaître la reprise de la croissance testiculaire liée au photopériodisme. 


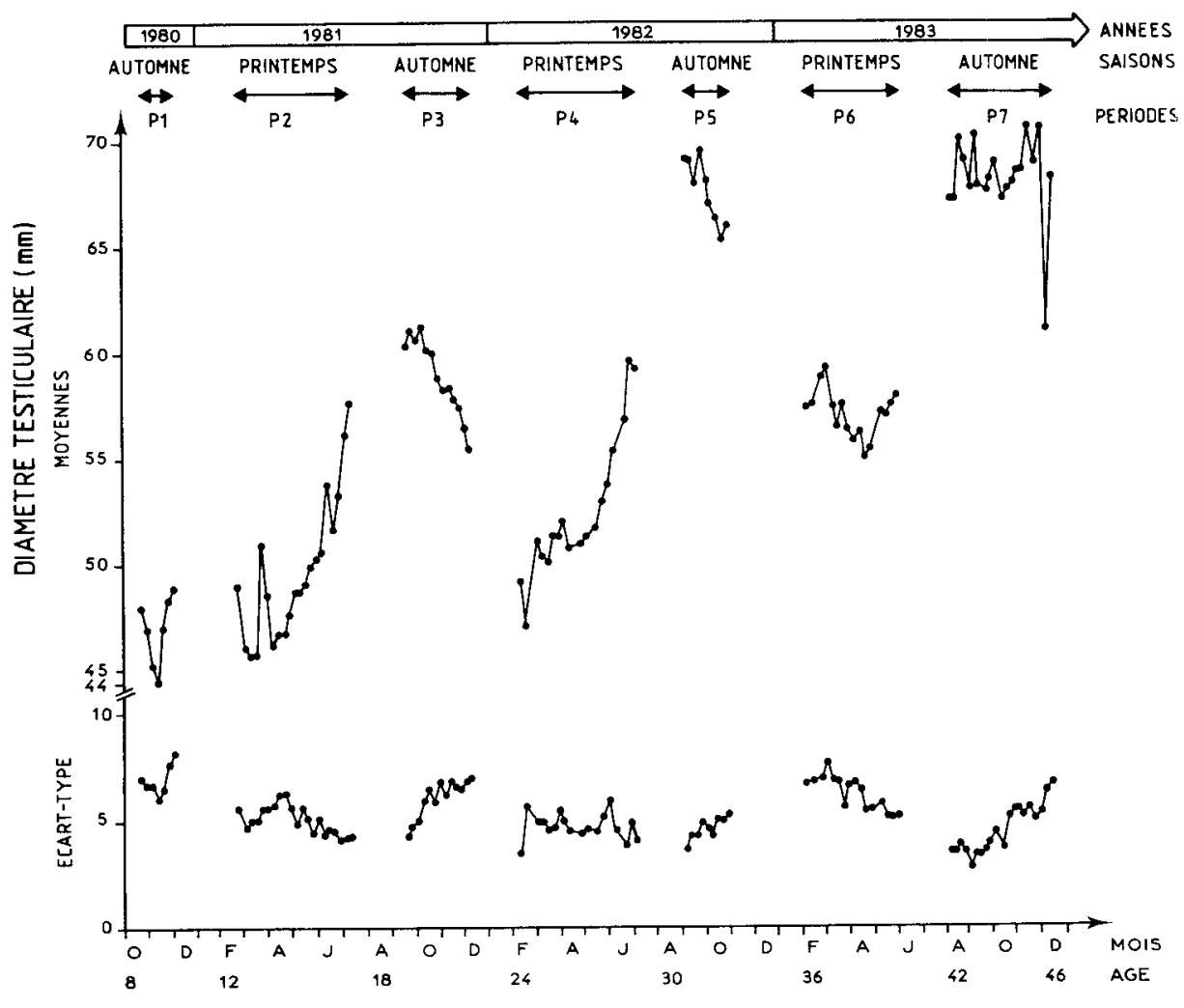

FIG. 1. - Variations hebdomadaires et saisonnières du diamètre testiculaire antéro-postérieur maximum (gonade + enveloppes) chez le bélier lle-de-France né au printemps ( $\mathrm{n}=17$ en $\mathrm{P} 1, \mathrm{n}=18$ de $\mathrm{P} 2$ à $\mathrm{P} 7)$.

On voit par ailleurs, que le DT augmente entre février 1982 et février 1983 (respectivement $49,2 \pm 5,2$ et $58,1+6,9 \mathrm{~mm}, \mathrm{P}<0,05$, moyenne des trois premières mesures de chaque mois) mais n'augmente plus entre mai 1982 et mai $1983(53,0 \pm 5,5$ et 57,1 $\pm 5,6 \mathrm{~mm}, P<0,05)$. La croissance testiculaire due au développement corporel s'achève donc entre février 1982 et mai 1982, c'est-à-dire lorsque les animaux ont entre 24 et 27 mois.

L'écart type de la moyenne est plus élevé pendant la $1^{r e}$ période qu'au cours des périodes 2 à 7 où il conserve à peu près la même valeur.

C) Anomalies morphologiques des spermatozoïdes (fig. 2). - En dehors des valeurs exceptionnellement élevées du mois d'août 1983 (P7) dues à de fortes températures (les températures maximales enregistrées sous abri par la Station Météorologique de Tours ont été supérieures à $30^{\circ} \mathrm{C}$ du 9 juillet au 16 juillet 1983), la proportion de formes anormales est toujours plus forte au printemps qu'en automne. 


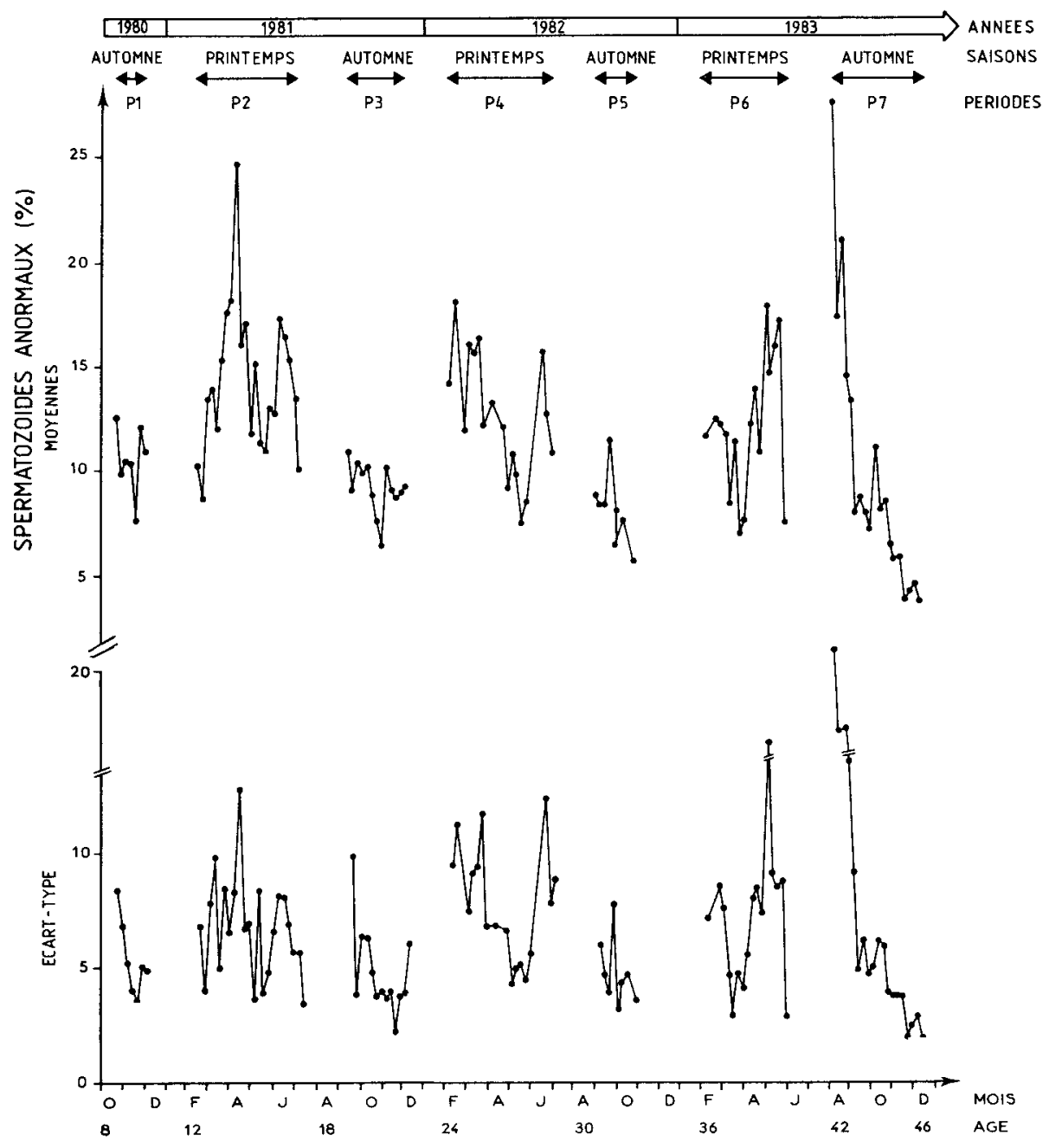

FIG. 2. - Variations hebdomadaires et saisonnières du pourcentage de spermatozoïdes anormaux chez le bélier l/e-de-France né au printemps ( $\mathrm{n}=17$ en $\mathrm{P} 1, \mathrm{n}=18$ de $\mathrm{P} 2$ à $\mathrm{P} 7)$.

La figure 2 et le tableau 1 montrent que cette proportion est maximale en février (P4) ou en mars-avril (P2, P6) selon les années mais que la qualité du sperme se dégrade à nouveau au mois de juin.

En automne les moyennes hebdomadaires restent faibles même chez l'agneau (P1) où elles ne varient qu'entre 7,7 et $15,7 \%$. 
TABLEAU 1

Pourcentages hebdomadaires maxima d'anomalies morphologiques (et leurs valeurs extrêmes) recensées dans le sperme au printemps chez le bélier /le-de-France $(\mathrm{n}=18)$.

\begin{tabular}{lccc}
\hline Période & Mois & \multicolumn{2}{c}{$\begin{array}{c}\text { Pourcentage maximum } \\
\text { observé }\end{array}$} \\
\hline P2 & Avril & 24,8 & $(8,0$ à 58,6$)$ \\
& Juin & 17,5 & $(5,3$ à 48,3$)$ \\
\hline P4 & Février & 18,3 & $(4,0$ à 48,6$)$ \\
& Juin & 15,8 & $(3,3$ à 48,6$)$ \\
\hline P6 & Avril & 18,1 & $(5,3$ à 62,3$)$ \\
& (non contrôlé en Juin) & & \\
\hline
\end{tabular}

( ) : pourcentages individuels extrêmes.

11. Relation entre les diamètres testiculaires moyens des mâles, ou entre leurs pourcentages moyens de spermatozoïdes, mesurés à deux périodes de leur vie.

A) Diamètre testiculaire. - Pour le calcul des corrélations entre périodes, les données ont été choisies comme suit:

- Première période : les deux mesures effectuées les $4^{\mathrm{e}}$ et $5^{\mathrm{e}}$ semaines.

- Périodes 2 à 7: (a) Au printemps, les trois valeurs les plus basses du mois d'avril qui est généralement considéré comme un mois de forte régression testiculaire chez l'lle-de-France. (b) En automne, les trois semaines où le DT moyen est le plus élevé. Cette triple mesure, effectuée à une époque de pleine activité sexuelle, permet, en effet, de se faire une idée assez précise des dimensions testiculaires maximales de la race.

En dehors de la quatrième période (tabl. 2), il existe toujours des corrélations significatives entre les valeurs trouvées chez l'agneau (P1) et chez l'adulte.

Au-delà de la première période (tabl. 2), les mesures faites pendant les mêmes saisons : $\mathrm{P} 2$ et $\mathrm{P} 4, \mathrm{P} 2$ et $\mathrm{P} 6, \mathrm{P} 4$ et $\mathrm{P} 6$ pour le printemps sont étroitement corrélées. Il en est de même pour l'automne entre P3 et P5, P3 et P7, P5 et P7 (non indiqué ici).

Ces liaisons sont en revanche beaucoup plus faibles et souvent non significatives lorsque les contrôles ont lieu à des saisons différentes : P2 et P3, P2 et P5, P2 et P7 (tabl. 2).

B) Anomalies morphologiques des spermatozoïdes. - Les anomalies étant peu nombreuses en automne chez l'adulte (P3, P5, P7), les corrélations entre P1 et P3, P1 et P5, P1 et P7 n'ont pas été calculées.

Les données ont été choisies de la manière suivante:

- $1^{\text {re }}$ période : les valeurs correspondant aux 5 premières mesures ;

- $2^{\mathrm{e}}$ période : les quatre pourcentages les plus élevés enregistrés en mars-avril ;

$-4^{\mathrm{e}}$ et $6^{\mathrm{e}}$ périodes : les valeurs relevées à des dates semblables. 
Corrélations simples, multiples et canoniques calculées entre les diamètres testiculaires mesurés à deux périodes de la vie des animaux $(\mathrm{n}=18)$.

\begin{tabular}{|c|c|c|}
\hline $\begin{array}{l}\text { Périodes } \\
\text { comparées }\left({ }^{1}\right)\end{array}$ & $\begin{array}{l}\text { Corrélations } \\
\text { simples }\left({ }^{2}\right)\end{array}$ & $\begin{array}{l}\text { Corrélations } \\
\text { canoniques }\left({ }^{2}\right)\end{array}$ \\
\hline \multicolumn{3}{|c|}{ I - Relation entre l'agneau et l'antenais ou l'adulte } \\
\hline $\begin{array}{l}\mathrm{P} 1-\mathrm{P} 2 \\
\mathrm{P} 1-\mathrm{P} 4 \\
\mathrm{P} 1-\mathrm{P} 6\end{array}$ & $\begin{array}{l}0,654^{* * *} \text { à } 0,747^{* * *} \\
\text { NS } \\
0,574^{* * *} \text { à } 0,580^{* * *}\end{array}$ & $\begin{array}{l}0,57^{* *}(2 \times 2, \quad 4 \& 5 \times 13 \& 11) \\
\text { NS } \\
0,48^{*}(2 \times 2,4 \& 5 \times 81 \& 80)\end{array}$ \\
\hline $\begin{array}{l}\text { P1-P3 } \\
\text { P1-P5 } \\
\text { P1-P7 }\end{array}$ & $\begin{array}{l}0,700^{* * *} \text { à } 0,817^{* * *} \\
0,574^{* * *} \text { à } 0,580^{* * *} \\
0,539^{* * *} \text { à } 0,668^{* * *}\end{array}$ & $\begin{array}{l}0,68^{* * *}(2 \times 2, \quad 4 \& 5 \times 31 \& 29) \\
0,63^{* * *}(2 \times 2, \quad 4 \& 5 \times 62 \& 60) \\
0,46^{*}(1 \times 2, \quad 4 \times 102 \& 101)\end{array}$ \\
\hline \multicolumn{3}{|c|}{ II - Relation chez l'animal de plus d'un an } \\
\hline $\begin{array}{l}\text { P2-P4 } \\
\text { P2-P6 } \\
\text { P4-P6 } \\
\text { P2-P3 } \\
\text { P2-P5 } \\
\text { P2-P7 }\end{array}$ & $\begin{array}{l}0,692^{* * *} \text { à } 0,821^{* * *} \\
0,719^{* * *} \text { à } 0,906^{* * *} \\
0,729^{* * *} \text { à } 0,875^{* * *} \\
\text { NS } \\
3 \text { coef. sign. sur } 9(0 \\
8 \text { coef. sign. sur } 9(0\end{array}$ & $\begin{array}{l}0,690^{* * *}(2 \times 2, \quad 14 \& 15 \times 52 \& 51) \\
0,88^{* * *}(2 \times 2, \quad 14 \& 15 \times 81 \& 80) \\
0,78^{* * *}(2 \times 2, \quad 50 \& 51 \times 81 \& 80) \\
\\
\left.0,541^{*}\right) \\
\left.0.768^{* * *}\right)\end{array}$ \\
\hline
\end{tabular}

$\left({ }^{1}\right)$ Les chiffres impairs représentent l'automne, les chiffres pairs le printemps.

$\left(^{2}\right)^{*}: P<0,05 ; *^{* *}: P<0,02 ;^{* * *}: P<0,01$.

Quelles que soient les périodes considérées (tabl. 3), les corrélations significatives (simples ou canoniques) sont peu nombreuses et leurs valeurs inférieures à celles obtenues pour le diamètre testiculaire. Toutefois, c'est entre la première et la seconde période c'est-à-dire lorsque l'animal est agneau et antenais, que la liaison est la plus étroite.

\section{Relations entre diamètre testiculaire et anomalies morphologiques des spermatozoïdes.}

Le calcul de ces relations a été fait à partir de l'ensemble de données obtenues de $\mathrm{P} 2$ à $\mathrm{P} 6$ incluses. Les résultats dépendent beaucoup du mode de calcul. Lorsque I'on raisonne sur les données individuelles, la corrélation (simple) est faible $\left(r=-261^{* *}, n=1212\right)$. Lorsque les calculs sont effectués sur les moyennes hebdomadaires du lot (18 mâles), on trouve une valeur beaucoup plus élevée et significative $\left(r=-0,671^{* *}, n=57\right)$.

\section{Relations trouvées au moment de l'abattage.}

II existe des relations hautement significatives entre : - le diamètre et le poids du testicule $\left(r=+0,890^{* * *}, n=2 \times 17\right)$; le diamètre testiculaire et le poids de l'épididyme $\left(r=+0,821^{* * *}, n=2 \times 17\right)$; - le poids du testicule et le poids de l'épididyme $\left(r=+0,867^{* * *}, n=2 \times 17\right) ;$ - le diamètre testiculaire pendant la $1^{\text {re }}$ période et au moment de l'abattage $(r=0,89, n=2 \times 17)$. 
TABLEAU 3

Corrélations simples et canoniques calculées à partir des données individuelles, entre les pourcentages de spermatozoïdes anormaux déterminés à deux périodes de la vie des animaux.

\begin{tabular}{|c|c|c|}
\hline $\begin{array}{l}\text { Périodes } \\
\left.\text { comparées ( }{ }^{1}\right)\end{array}$ & $\begin{array}{l}\text { Corrélations } \\
\text { simples }\left({ }^{2}\right)\end{array}$ & $\begin{array}{l}\text { Corrélations } \\
\text { canoniques }\left({ }^{2}\right)\end{array}$ \\
\hline & \multicolumn{2}{|c|}{ I - Relation entre l'agneau et l'adulte $(n=17)$} \\
\hline \multirow[t]{2}{*}{$\begin{array}{l}\mathrm{P} 1-\mathrm{P} 2 \\
\mathrm{P} 1-\mathrm{P} 4 \\
\mathrm{P} 1-\mathrm{P} 6\end{array}$} & $\begin{array}{l}6 \text { coef. sign. sur } 20\left(0,477^{*} \text { à } 0,736^{* * *}\right) \\
4 \text { coef. sign. sur } 20\left(0,606^{* * *} \text { à } 0,674^{* * *}\right) \\
2 \text { coef. sign. sur } 20\left(0,507^{*} \text { à } 0,643^{* * *}\right)\end{array}$ & $\begin{array}{l}0,70^{* * *}(5 \& 2 \times 15 \& 18) \\
0,55^{* *}(5 \& 4 \times 48 \& 51) \\
0,46^{*}(5 \& 2 \times 72 \& 73)\end{array}$ \\
\hline & \multicolumn{2}{|c|}{ II - Relation chez l'animal adulte $(n=18)$} \\
\hline $\begin{array}{l}\text { P2-P4 } \\
\text { P2-P6 } \\
\text { P4-P6 }\end{array}$ & $\begin{array}{c}1 \text { coef. sign. sur } 20\left(0,502^{*}\right) \\
3 \text { coef. sign. sur } 20\left(0,481^{*} \text { à } 0,648^{* * *}\right) \\
\text { NS }\end{array}$ & $\begin{array}{c}\text { NS } \\
0,76^{* * *}(14 \& 16 \times 76 \& 73) \\
\text { NS }\end{array}$ \\
\hline
\end{tabular}

(1) Les chiffres pairs représentent le printemps, les chiffres impairs l'automne.

(2) ${ }^{*}: \mathrm{P}<0,05 ;{ }^{* *}: \mathrm{P}<0,02 ;{ }^{* * *}: \mathrm{P}<0,01$.

\section{Discussion.}

Pour pouvoir répondre aux questions posées, il était nécessaire d'observer les animaux pendant un temps suffisamment long. C'est la raison pour laquelle notre étude a duré plusieurs années.

\section{Evolution générale.}

A) Diamètre testiculaire. - La forte corrélation entre le diamètre et le poids du testicule, proche de celles obtenues par Colyer (1971), prouve que les mesures faites sur l'animal vivant pendant les sept périodes, reflètent d'assez près l'évolution pondérale réelle de la gonade.

Les variations saisonnières du diamètre testiculaire sont, elles aussi, conformes aux données de la littérature [Ortavant (1958, Ile-de-France); Schanbacher (1979, Suffolk); Lincoln (1979, Suffolk); Colas et al., (1986, Vendéens); etc...] ; régression vers la fin de l'automne (novembre), reprise en jours longs (fin mai, début juin). Nos béliers expérimentaux avaient donc une évolution sexuelle tout à fait conforme à celle de la race.

On peut remarquer, enfin, que la croissance de la gonade s'achève à l'âge d'environ deux ans, c'est-à-dire à peu près au même moment que le développement corporel (Alberio, 1976).

Quant à l'écart type, il conserve à peu près toujours la même valeur au cours des différentes périodes. Le coefficient de variation qui reflète l'importance relative des différences individuelles, est donc plus élevé au printemps qu'en automne et diminue au fur et à mesure que l'animal vieillit. 
B) Anomalies morphologiques des spermatozoides. - La dégradation de la qualité des gamètes, que nous observons au printemps (février à avril) pendant trois années consécutives, a déjà été signalée chez d'autres races: Suffolk (Schanbacher, 1979), Finnoise (Amir et al., 1986), Vendéenne (Colas et al., 1986). Elle apparaît, en outre, au moment où le diamètre testiculaire est minimum. Formes anormales et dimensions de la gonade évoluent (fig. 1 et 2) donc en sens inverse comme l'indique la corrélation $(r=-0,671)$ entre leurs moyennes hebdomadaires respectives. Celle-ci est cependant beaucoup plus faible (mais toujours significative), lorsque les calculs sont faits à partir des données individuelles. Cette contradiction apparente peut s'expliquer par le fait que chez un même individu, les variations hebdomadaires du pourcentage d'AM au printemps sont beaucoup plus fortes que celles du diamètre testiculaire et que les valeurs les plus faibles peuvent apparaître à des moments différents d'une année à l'autre.

II est surprenant de rencontrer, au mois d'août 1983 (P7) autant de spermatozoïdes anormaux et des écarts individuels aussi élevés. Ces malformations sont en fait, de nombreuses études l'ont montré [Dutt et Hamm (1957) : Howarth (1969); Smith (1971); Rathore $(1968,1970)]$, la conséquence des fortes températures ambiantes enregistrées quelques semaines auparavant. Une analyse plus détaillée (non rapportée ici) de nos résultats nous a montré qu'il s'agissait surtout de défauts localisés au niveau de la tête, caractéristiques d'un stress thermique (Rathore, 1968, 1970). Il faut attendre la fin du mois et même, chez les animaux les plus perturbés, la dernière quinzaine de septembre pour retrouver chez tous les béliers un profil normal. Les mêmes températures peuvent donc entraîner des effets différents selon les individus.

Contrairement à ce que l'on admet en général (Louw et Joubert, 1964 ; Skinner et Rowson, 1968; Colas et Zinszner, 1975), les anomalies sont peu nombreuses chez l'agneau dans notre expérience. Cette discordance tient à l'âge des animaux (8-9 mois), à la saison de collecte (automne) et à l'époque de naissance (printemps). Toutefois, des examens réalisés à un stade plus précoce (6 mois) auraient sans doute permis de déceler un plus grand nombre de malformations cellulaires et peut-être aussi une plus forte variabilité individuelle.

\section{Relations entre périodes.}

A) Dimensions testiculaires. - L'un des résultats intéressants de cette expérience réside dans la mise en évidence d'une relation étroite entre les moyennes enregistrées en avril sur des antenais et celles que l'on relève sur les mêmes animaux 12 ou 24 mois plus tard. Dans une telle étude, les corrélations canoniques donnent toujours plus d'information que les corrélations simples puisqu'elles sont établies non pas à partir d'un couple de données mais à partir de deux groupes de mesures. On voit par exemple (tabl. 2) que 69 à $88 \%$ de la variabilité existant en P4 ou en P6 provient de celle que l'on trouve en P2. Choisir au printemps (avril), parmi les béliers antenais, ceux dont les testicules sont les plus développés revient à créer une sous-population dont la variabilité ultérieure sera réduite de 69 à $88 \%$. En procédant ainsi, on peut repérer très tôt les mâles 
qui sont les moins sensibles à l'augmentation de la durée d'éclairement, c'est-à-dire les plus forts producteurs de semence. Une telle «sélection » revêt évidemment un grand intérêt zootechnique.

Les relations sont en revanche beaucoup plus lâches entre les mesures que l'on peut faire sur les mêmes animaux au printemps et en automne. Les relevés effectués sur un bélier pendant la saison sexuelle ne donnent donc aucune indication précise sur son évolution testiculaire au printemps.

En dehors de la $4^{\circ}$ période et malgré la faiblesse de l'effectif expérimental, nous avons trouvé des corrélations très significatives entre les données observées pendant la $1^{\text {re }}$ période et pendant les périodes ultérieures. Les dimensions testiculaires d'un sujet jeune et du même sujet adulte ne sont donc pas indépendantes. II est important de savoir qu'à partir de deux mesures faites à la mi-automne, on peut dès la première année réduire de plus de la moitié la variabilité qui existera entre ces animaux 6 ou 12 mois plus tard. Cette relation existe aussi après abattage puisque la largeur du scrotum mesurée à l'âge de 8 mois et demi est fortement corrélée au poids du testicule 38 mois plus tard (46 mois). On peut donc effectuer chez l'agneau une présélection précoce qui aura pour effet de réduire fortement l'hétérogénéité ultérieure.

B) Anomalies morphologiques des spermatozoïdes. - II est surprenant d'observer aussi peu de relation chez l'adulte entre les différents contrôles opérés au printemps, à 1 ou 2 ans d'intervalle. Ce manque de similitude vient, comme nous l'avons dit précédemment, du fait que les variations hebdomadaires individuelles de la qualité du sperme peuvent être très fortes et qu'elles n'ont pas lieu chaque année aux mêmes moments. Deux ou trois contrôles au mois d'avril ne sont donc pas suffisants pour permettre de juger l'aptitude d'un géniteur à produire du sperme de bonne qualité au printemps. Sans doute faut-il allonger la période de contrôle.

II existe, par contre, un lien étroit entre la proportion d'anomalies recensées dans le sperme d'un agneau de 8,5 mois en automne et celle que l'on trouvera chez le même animal 6 mois plus tard.

On peut donc, là aussi, opérer de bonne heure, un certain choix parmi les jeunes reproducteurs. II serait évidemment intéressant de vérifier cette relation sur d'autres races en pratiquant des contrôles plus précoces, dès l'âge de 6 mois. On peut supposer que les mêmes observations faites sur des agneaux nés au printemps permettront de prévoir avec plus de précision leur devenir à la même saison.

\section{Conclusion.}

Le diamètre testiculaire et le pourcentage de spermatozoïdes anormaux, mesurés chaque semaine, sur un ensemble de béliers lle-de-France nés au printemps, évoluent en sens inverse au cours de l'année. Lorsque les animaux sont pris individuellement, toutefois, cette relation est moins étroite tout en restant significative. 
Au mois d'avril, il existe de très fortes corrélations entre les valeurs testiculaires prises à 12 ou à 24 mois d'intervalle. II est donc possible, chez des sujets antenais de même âge, nés au printemps, de réaliser à cette saison une pré-sélection précoce fondée sur le plus ou moins grand développement de la gonade.

Les corrélations sont en revanche très faibles entre l'automne et le printemps.

On trouve très peu d'analogie entre les observations morphologiques effectuées au printemps sur le sperme des mêmes béliers à une ou deux années d'écart et à des dates à peu près identiques.

Les dimensions testiculaires d'un agneau de 8 mois et du même animal adulte sont fortement corrélées, en particulier en automne. Leur mesure permet donc de trier les meilleurs producteurs de semence dès l'âge de 8 mois.

La même démarche peut difficilement être appliquée à la qualité du sperme puisque les corrélations sont, dans l'ensemble, plus faibles.

Reçu en septembre 1987.

Accepté en janvier 1988.

Remerciements. - Les auteurs tiennent à remercier Monsieur R. Ortavant pour ses commentaires et le secrétariat qui a assuré la frappe de ce texte.

\section{Références}

ALBERIO R., 1976. Rôle de la photopériode dans le développement de la fonction de reproduction chez l'agneau lle-de-France, de la naissance à 21 mois. Th. Doct. $3^{e}$ cycle, Univ. Paris VI, $57 \mathrm{pp}$

AMIR D., GACITUA H., RON M., LEHRER A. R., 1986. Seasonal variation in semen characteristics and the fertility of Finn Cross rams subjected to frequent ejaculations. Anim. Reprod. Sci., 10 , $75-84$.

COLAS G., 1980. Variations saisonnières de la qualité du sperme chez le bélier lle-de-France. 1. Etude de la morphologie cellulaire et de la motilité massale. Reprod. Nutr. Dévelop., 20, $1789-1799$

COLAS G., 1981. Variations saisonnières de la qualité du sperme chez le bélier lle-de-France. 2. Fécondance : relation avec les critères qualitatifs observés in vitro. Reprod. Nutr. Dévelop., 21, 399-407.

COLAS G., ZINSZNER F., 1975. Production spermatique et développement testiculaire chez l'agneau de race lle-de-France et Préalpes. $1^{\text {res }}$ Journées de la Recherche Ovine et Caprine, Les Races prolifiques (ITOVIC, Ed.), pp. 235-243.

COLAS G., GUERIN Y., LEMAIRE Y., MONTASSIER Y., DESPIERRES J., 1986. Variations saisonnières du diamètre testiculaire et de la morphologie des spermatozoïdes chez le bélier Vendéen et chez le bélier Texel. Reprod. Nutr. Dévelop., 26, 863-875.

COLYER R. J., 1971. Development of the testis and epididymis of the Clun Forest ram. J.agric. Sci. Camb., 76, 433-441.

COUROT M., de REVIERS M. M., PELLETIER J., 1975. Variations in pituitary and blood LH during puberty in the male lamb. Relation to time of birth. Ann. Biol. anim. Bioch. Biophys., 15, 509-516.

DUTT R. H., HAMM P. T., 1957. Effect of exposure to high environmental temperature and shearing on semen production of rams in winter. J. anim. Sci., 16, 328-334.

HOWARTH B., 1969. Fertility in the ram following exposure to elevated ambient temperature and humidity. J. Reprod. Fert., 19, 179-183. 
LEFEBVRE J., 1976. Introduction aux analyses statistiques multidimensionnel/es, $275 \mathrm{p}$. Masson Edit.

LINCOLN G. A., 1977. Changes in pituitary responsiveness to luteinizing hormone releasing hormone in rams exposed to artificial photoperiods. J. Endocrinol., 73, 519-527.

LINCOLN G. A., 1979. Photoperiodic control of seasonal breeding in rams. The significance of short days refractoriness. In : Proc. 6th int. Congr. Endocrinol., Melbourne, pp. 431-441.

LOUW D. F., JOUBERT D. M., 1964. Puberty in the male Dorper sheep and Boer goat. South Afric J. agric. Sci., 7, 509-520.

NEVES J. P., GÜNZEL A. R., SCHMIDT H., 1980. Einfluss der Jahreszeit auf der Sperma Qualität von Merino Fleischschafen. Zuchthygiene, 15, 118-125.

ORTAVANT R., 1958. Le cycle spermatogénétique chez le bélier. Th. Doct. ès Sci., Paris, 127 pp., $N^{\circ}$ A 3118-0 3990.

ORTAVANT R., THIBAULT C., 1956. Influence de la durée d'éclairement sur la production spermatique du bélier. C.R. Soc. Biol., 150, 358-361.

PELLETIER J., 1971. Influence du photopériodisme et des androgènes sur la synthèse et la libération de $L H$ chez le bélier. Th. Doct. Etat ès Sci. nat. Paris $\mathrm{N}^{\circ}$ A 5441.

RATHORE A. K., 1968. Effects of high temperature on sperm morphology and subsequent fertility in Merino sheep. Proc. austr. Soc. anim. Prod., 7, 270-274.

RATHORE A. K., 1970. Fertility of rams heated for 1, 2, 3 and 4 days, mated to superovulated ewes. Austr. J. agric. Res., 21, 355-358.

REVIERS M. de, HOCHEREAU de REVIERS M. T., BLANC M. R., BRILLARD J. P., COUROT M., PELLETIER J., 1980. Control of Sertoli and germ cell populations in the cock and sheep testes. Reprod. Nutr. Develop., 20, 241-249.

SCHANBACHER B. D., 1979. Increased lamb production with rams exposed to short daylengths during the non-breeding season. J. anim. Sci. 49, 927-932.

SCHANBACHER B. D., FORD J. J., 1979. Photoperiodic regulation of ovine spermatogenesis: relationship to serum hormones. Biol. Reprod., 20, 719-726.

SKINNER J. D., ROWSON L. E. A., 1968. Puberty in Suffolk and cross-bred rams. J. Reprod. Fert., 16, 479-488.

SMITH J. F., 1971. The effect of temperature on characteristics of semen of rams. Austr. J. agric Res., 22, $481-490$. 